\title{
The Approach to Automatic Detection of the Content of the Smart Electricity Meter LCD Cycle Display
}

\author{
Wen-jia CAl ${ }^{1, a}$, Zhi-yao ZHENG ${ }^{2, b}$, Fan LI $^{3, c}$, YUAN WANG ${ }^{4, d}$ \\ ${ }^{1}$ State Grid Hubei Electric Power Research Institute, China \\ ${ }^{2}$ State Grid Wuhan Electric Power Supply Company, China \\ ${ }^{3}$ State Grid Hubei Electric Power Research Institute, China \\ ${ }^{4}$ State Grid Hubei Electric Power Research Institute, China \\ aemail, bemail, ${ }^{c}$ email, ${ }^{\mathrm{d}}$ email
}

Keywords: smart electricity meter, cycle display, LCD display and detection, image processing

\begin{abstract}
This paper is a study of the approach to automatic detection of the content of the smart electricity meter LCD cycle display and signal lights. By this approach, in the LCD liquid crystal display detection unit of the smart electricity meter detection line, the electricity meter can be switched to a certain designed display status via RS485 communication interface and the specific protocol directions, so as to be compared with the pictures taken by smart cameras to verify the qualification of the content of the smart electricity meter LCD cycle display and signal lights. This approach realizes the detection of LCD liquid crystal display of the smart electricity meter with simple hardware and speedy detection, providing the present detection with better accuracy, advance and efficiency.
\end{abstract}

\section{Introduction}

The smart electricity meter detection line has been popularized and applied domestically, which realizes the automatic detection of smart electricity meters in large quantity. The outside detection unit, which is composed of industrial cameras, professional light and professional image processing software, plays an significant and operative part in the automatic detection system[1-2]. By starting the LCD background light and full-screen display of the smart electricity meter, the photos can be taken automatically of the outlook of the smart electricity meter, of the signals and logos, and of the full screen of the LCD display. All of the above can be compared with the pre-designed standard scheme, in order to detect the electricity meter LCD liquid crystal display[3-4]. Whereas the cycle display and the signal lights under running state are not been detected in the present domestic applied outlook detection unit, in order to ensure the thorough detection of smart electricity meters and the correct status reflection of the signal lights, it is indispensable to study an approach to the automatic detection of the content of the smart electricity meter LCD cycle display and signal lights.

\section{The Traditional Detection of LCD Display of Electricity Meter}

The traditional full-screen detection is achieved by image recognition module, starting the LCD background light and full-screen display of the smart electricity meter and automatically completing the image collection of the outlook of the electricity meter, signals and logos, and the full-screen LCD display, which can be processed by smart image software, to realize the automatic detection of flaws of the panel and full screen, like Fig.1: 


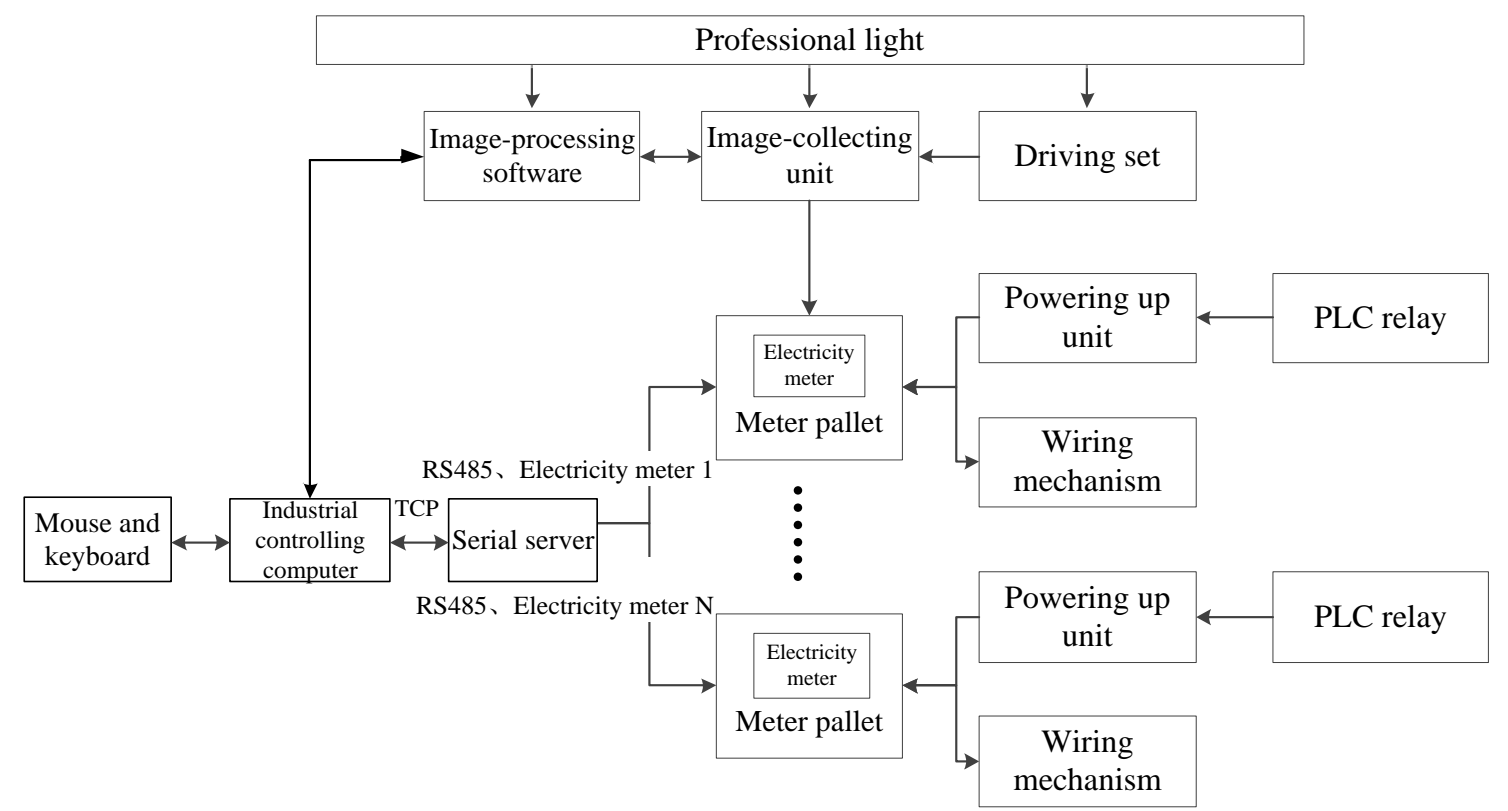

Fig. 1, Traditional LCD liquid crystal display (LCD) detection scheme

The traditional detection only takes photo and make comparison when there is full-screen LCD display, and the detection of smart electricity meters is limited to missing segment, shortcut and blurred screen of the LCD display of electricity meters, which can not need the demand of the realization of automatic detection of the cycle display and operation status of electricity meters.

\section{The Significance of Detection of LCD Cycle Display of Electricity Meter}

Various parameters of smart electricity meters can be displayed in LCD screen of smart electricity meters, of which the content can be controlled by programming[5]. The purpose of cycle display detection is to see whether the content of each screen of smart electricity meters meets the demand of State Grid Smart Electricity Meter Technological Regulations. Through the simulation of on-site operation malfunction, the detection program can detect whether there is corresponding malfunction reminder, disorder signal or alarming lights, which is profoundly significant to openness, equality and fairness of smart metering.

\section{The New LCD Cycle Detection of Electricity Meter}

\subsection{System Design of LCD Cycle Detection Unit of Electricity Meter}

In the detection system of smart electricity meters, to fulfill the detection function of LCD cycle display of the electricity meter without increasing fault points or affecting detection tempo, a systematic approach to automatic detection of the content of the smart electricity meter LCD cycle display is proposed to make the detection simple, advanced and efficient.

Via setting up the additional switching module in the general controlling center, and applying RS485 communication interface and the specific protocol directions to the smart electricity meters, the system can make the smart electricity meter switch to the pre-designed status, in which the content of the screen display can meet all the demand of State Grid Smart Electricity Meter Technological Regulations on screen display. The purpose of cycle display detection is served by industrial camera shooting and image processing. The necessary hardware includes industrial computer, serial server, image collecting unit, driving set, powering up unit, wiring mechanism, PLC relay, professional lights, etc.

By using the RS485 communication interface of smart electricity meter, via the specific directions transmitted from serial server, the system controls the electricity meter to switch to a pre-designed 
display status. The industrial computer communicates with serial server via Ethernet, collects on-site statistics and processes images, and compares the collected statistics with the standard ones, so as to discern the correctness of the content and to acknowledge the function of the signal lights, which is to judge the qualification of the electricity meter. The driving set is a transformer or servomechanism, controlling the position of image-collecting unit, to make sure the industrial camera moving back and forth to take photos of the smart electricity meters in different working positions in the planned track. The powering up unit and PLC relay function in parallel, to control the on and off of the electricity meters, as well as the connection and disconnection of the wiring structure.

The general scheme of outlook detection is shown in Fig. 2:

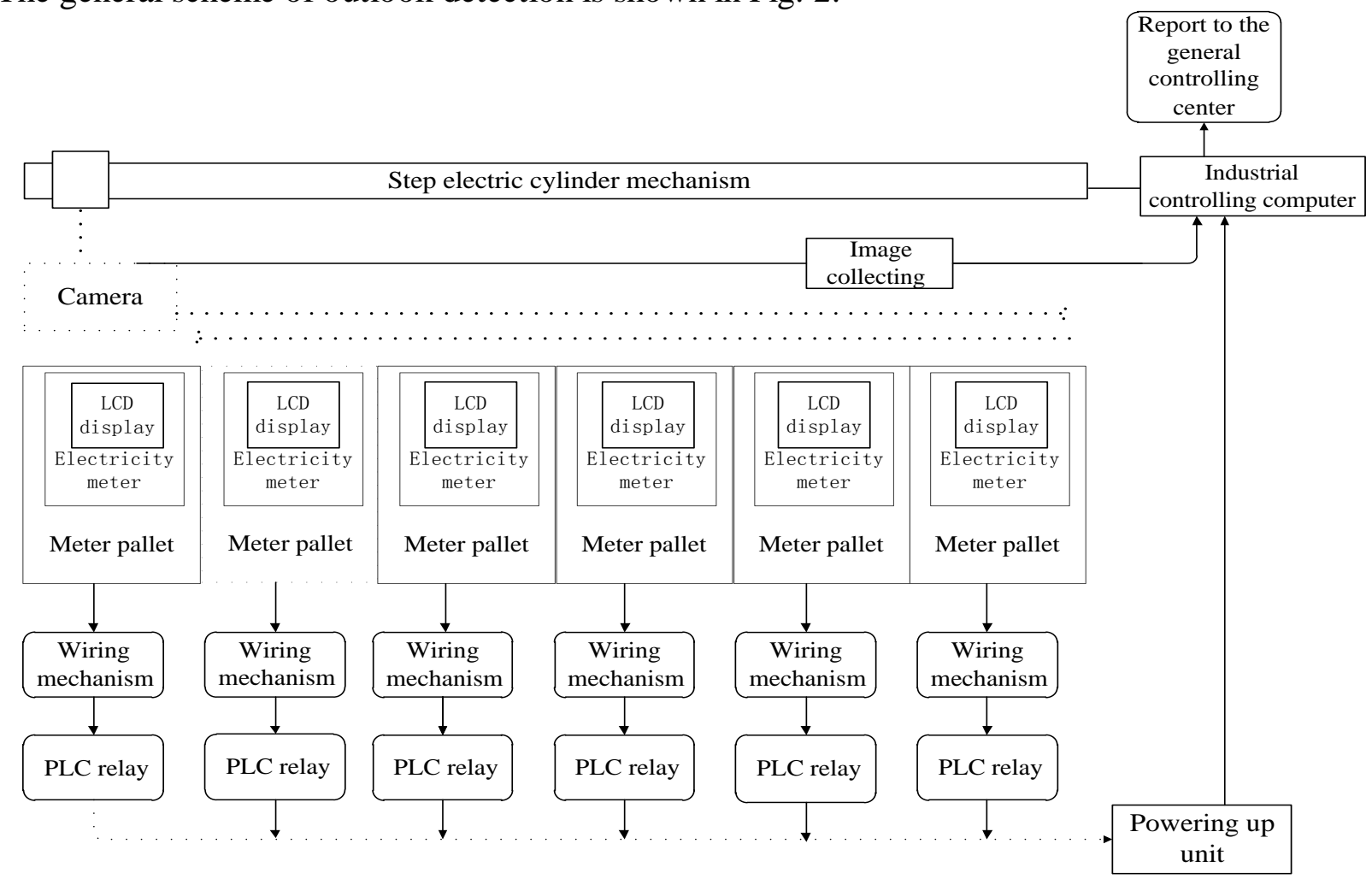

Fig. 2, Schematic diagram of the overall solution for LCD display and detection system

\subsection{Design Plan of Cycle Display Function Hardware}

The general controlling center is connected to multiple smart electricity meters and their RS485 communication interface, each of which can perform communication in parallel with others. The serial server consists of the general controlling center and the converter. The former is connected to the latter with CAN general wire, and communicates with smart electricity meter via RS485. The realization principal of cycle display function hardware is shown in Fig. 3:

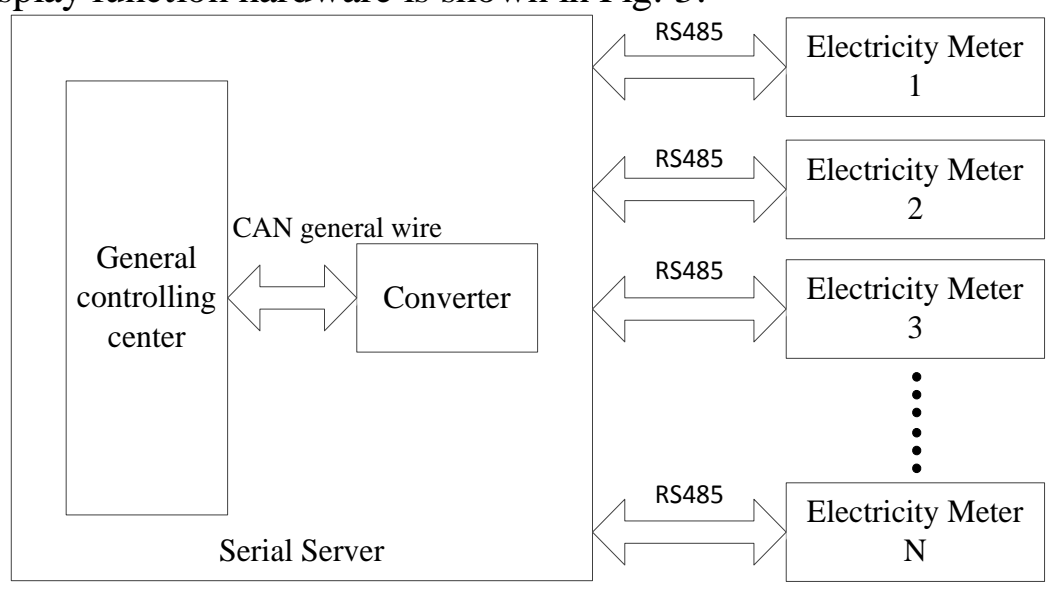

Fig. 3, LCD cycle display function hardware design schematic 
The advantage of this plan lies in the simple structure with convenient plugging of the hardware, the uniform of the screen shift without pressing buttons by the robot to shift screens, and the automatic completion of the detection. The precision can be up to $100 \%$, with the misjudgment below $1 \%$. Each photo-collecting takes no more than 3 seconds and with the high-speed processing, the system can synchronize the detection of multiple smart electricity meters.

\subsection{The Option of Screen Shifting Orders of RS485}

In accordance with DL/645-2007, smart electricity meter supports LCD view of orders, which is to read. Data domain contains data sign and serial number of the data item ready to be viewed, and when the data sign and serial number are both FF, there displays the full screen. LCD order view display and the background lights last 10 seconds. The content of the data item viewed is displayed on the LCD screen. When the power is up again, it will retreat from the status mentioned above, and goes into the first screen of cycle display.

The survey in multiple electricity meter providers shows that both single-phase meter and triphase meter support the data item of automatic cycle display and bottom cycle display in State Grid Smart Electricity Meter Technological Regulations, as well as the full-screen display.

\subsection{Design Plan of Cycle Display Function Software}

The Approach to automatic detection of the content of the smart electricity meter LCD cycle display can be divided into the following steps:

\section{(1) Wiring and Powering-up}

When the test of smart electricity meter LCD display starts, wiring structure operates on the smart electricity meter, meanwhile the PLC relay pulls in, and the powering-up unit wakes up the smart electricity meters, so the screen is lighted.

\section{(2) Image Collecting}

Image-collecting unit mainly contains the smart industrial camera. The camera moves towards the electricity meters in the planned track, when it fits in the right position, it takes photos of the full screen of the electricity meters, collects the on-site data and transmits the data to the industrial computer for information managemen $\mathrm{t}$.

\section{(3) Image Processing}

Image-collecting data is processed by image-processing software. The photos of each screen taken in the first pre-detection of the smart electricity meters can be regarded as the standard images, which can be used in the comparison with the automatically-taken crystal liquid initial photos, to find the disqualified items like non-displaying, segment missing and damaging. All the data can be sent to the industrial controlling computer for comprehensive data management.

\section{(4) Cycle Display of Shifting Screen}

When the pre-detection is completed, the system automatically transmits shifting-screen orders via RS485 communication interface of the smart electricity. Image-collecting unit collets the on-site data to compare with the standard data, and transmits the compared data to the industrial controlling computer for comprehensive data management. The same approach can be applied to the image collecting and processing until the detection of the last screen.

\section{(5) The Completion of the Pallet Detection}

When all the programs of the smart electricity meter detection are completed in the current pallet, the detection order for the next pallet will be sent to the system to perform the detection of the electricity meters. This process goes on until hype completion of the last pallet detection.

\section{(6) The End of the Detection Mission}

With the detection of all the electricity meters in the detective unit coming to the end, the detection device automatically power-off the smart electricity meters, and the camera goes back to its original position. All the on-site data and compared results are uploaded to the general controlling center of the serial server for comprehensive management. Mission accomplished. 


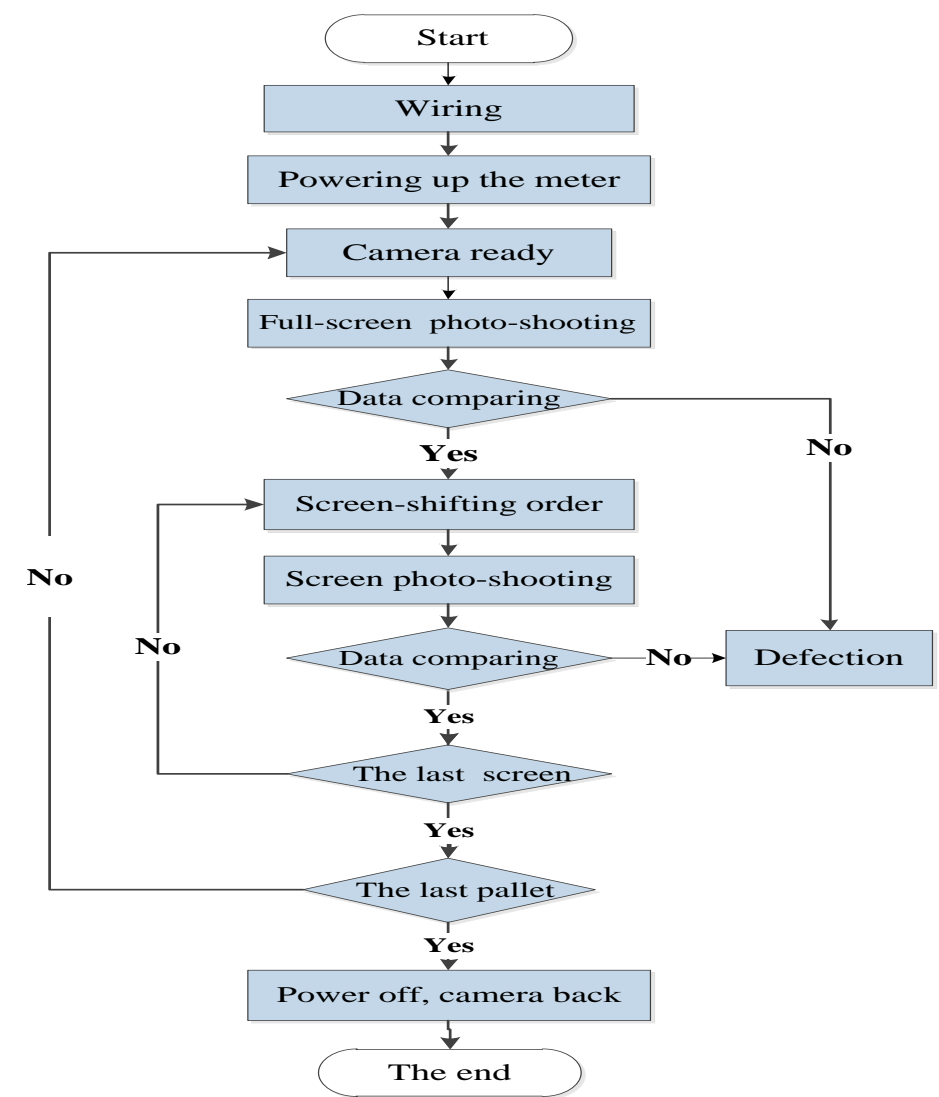

Fig. 4, LCD cycle display function software design schematic

\section{Conclusions}

An approach to automatic detection of the content of the smart electricity meter LCD cycle display is posted in this paper, according to the unicity of LCD display detection in the outlook detection unit of smart electricity meter detection system. This approach not only realizes the synchronized detection of multiple meter positions, but also solves the problem of the traditional method, i.e. Only the full screen of LCD detection can be performed, but the other parameters are neglected. In the paper, the 99 items of parameter content appointed in the smart electricity meter technological regulations are shown through automatic screen-shifting. Each of the parameters can appear in turns and each screen can be photo-shot for recognition. The on-site data collecting is accurate and convenient, significantly lowers the reject rate. This approach is technologically advanced with creatively new schemes.

\section{References}

[1] XIAO TAO, ZHENG FAN. Application Research of Quality Monitoring Technique for Automatic Metrological Verification Pipeline System [J]. Electrical Measurement \& Instrumentation, 2013, 50:72-76.

[2] ZHANG YAN, HUANG Jin-juan. Research and Application of Intelligent Verification Line System for Electric Energy Meter [J]. Electrical Measurement \& Instrumentation, 2009, 46(12):73-77.

[3] HE Zhi-qiang, DING TAO, XU Er-qiang, YANG Nai-gui ,YU Yi-zhou, ZHANG WEI. Application of PalMax and PalQuick in Smart Electricity Meter's LCD Detection. Automation \& Instrumentation. 2012.12.18

[4] HNEPC Metering Center, SMS Electric Co.,Ltd Zhengzhou. A LCD Screen Display Detection System of Smart Electric Meter [P]. Patent No.: 201120421737.2

[5] Q/GDW1356-2013 The Type Specification for Smart Triphase Electricity Meters. 